\title{
Infracciones de tráfico de los jóvenes suecos después de consumir alcohol*
}

\author{
BORSCHOS, B. \\ Centre for Social Research on Alcohol and Drugs (SoRad). Stockholm University (Sweden) \\ Enviar correspondencia: \\ Björn Borschos. bjorn.borschos@bra.es
}

\section{RESUMEN}

El presente estudio señala la existencia de un verdadero descenso en el número de infracciones cometidas por los jóvenes conductores suecos bajo los efectos del alcohol, especialmente durante los años noventa. Tras analizar las posibles causas del mismo, se llega a la conclusión de que la explicación más plausible de este fenómeno residiría en la estrategia preventiva aplicada en el país, basada en una política contra el alcohol muy estricta (la policía tiene amplios poderes, las infracciones son severamente castigadas, el límite de alcohol tolerado para conducir muy bajo...), política apoyada por un amplio sector de los ciudadanos y conocida como el 'modelo escandinavo'. El autor ha mostrado que es posible, con la ayuda de la legislación y de las campañas informativas, mitigar uno de los problemas más graves relacionados con el consumo de alcohol. La integración de Suecia en la Unión Europea está teniendo, sin embargo, importantes consecuencias disruptoras en su política contra el alcohol.

\begin{abstract}
This study indicates that there has been a real decline in the number of reported drink-driving offences committed by youths in Sweden, especially during the 90's. It analyses why and comes to the conclusion that the most plausible explanation for this decline lies in the application in the country of a successful preventive strategy, based on a tough alcohol policy (the police have extensive powers, punishments for offences are severe, drink-driving limits low...) supported and backed by the general public and often described as the Scandinavian model. The author has shown that it is possible by means of legislation and information campaigns to reduce one of the most serious alcohol-related problems. However, Sweden's membership of the European Union has had important implications with regard to the alcohol policy pursued in this country.
\end{abstract}

Key words: alcohol, driving, Sweden, young people, preventive policies, Scandinavian model.

Palabras clave: alcohol, conducción, Suecia, jóvenes, estrategias preventivas, modelo escandinavo.

S uecia ha realizado importantes inversiones destinadas a evitar que la gente conduzca tras ingerir alcohol ${ }^{1}$, incrementando así la seguridad de sus carreteras. Todos los esfuerzos se basan en la clara relación, tanto a nivel individual como nacional, entre el consumo de alcohol y el riesgo de sufrir un accidente de tráfico.
A nivel individual, este riesgo aumenta incluso cuando existe una baja concentración de alcohol en la sangre (Andréasson \& Jones, 1999; Golderg, 1970) y se incrementa exponencialmente al aumentar ésta (BAC). Por ejemplo, el riesgo de sufrir un accidente que afecte a un solo coche se duplica virtualmente al aumentar un 0,02\% el nivel de alcohol en sangre

\footnotetext{
* Publicado previamente en Nordic Studies on Alcohol and Drugs Vol17, English suplement (2000) pp.43-56, con el título 'Drink -driving offences among Swedish youths. Recent trends in development".

Conducir bajo los efectos del alcohol es un delito regulado por los párrafos 4 y $4^{a}$ del Acta (1951:649) que castiga algunas infracciones de tráfico. La ley dice que el conductor de un vehículo de motor que tiene una concentración de alcohol en la sangre de al menos 0,02\% o una concentración de alcohol semejante en el test de alcoholemia, o que no es capaz de manejar con seguridad dicho vehículo, es culpable del delito de conducir ebrio. Una consideración clave para determinar el agravamiento de la culpa es que la concentración de alcohol exceda el $0,1 \%$. También se tiene en cuenta si el conductor ha ingerido grandes cantidades de alcohol u otras sustancias, o si ha constituido una seria amenaza para la seguridad vial. El castigo prescrito para esa clase de infracciones es una multa o una sentencia de un máximo de seis meses de cárcel. En los casos más graves la sentencia puede llegar a ser hasta de dos años.
} 
(Zador, 1991). Asimismo, es un hecho probado que el porcentaje de conductores que han consumido alcohol tiende a ser mayor en los accidentes más graves (Ross, 1982). Östrom y Eriksson (1993) han demostrado que en más de la mitad de los accidentes mortales en los que se ve envuelto un solo coche, el conductor había ingerido previamente alcohol.

Las investigaciones ponen, además, de manifiesto que cualquier variación en el consumo total de alcohol incide en el número de accidentes de tráfico: cuando aumenta dicho consumo, los accidentes suelen ser más numerosos (BRA, 1998; Norström \& Andersson, 1996). Diversos estudios han mostrado la existencia de una asociación similar entre el consumo de alcohol y las infracciones de tráfico registradas (BRA, 1998; Smart \& Mann, 1987; Kendell, 1984).

Pero el alcohol no es el único factor de riesgo en los accidentes de tráfico. Otro factor de enorme importancia es la edad del conductor. Los jóvenes que acaban de obtener el permiso de conducir tienen un riesgo ocho o diez veces mayor de sufrir un accidente que los conductores con más edad y experiencia (Spolander, 1992). Ello se debe no sólo a la falta de experiencia (Gregersen, 1997), sino también a la tendencia a sobrevalorar su habilidad (Gregersen, 1996). El resultado de todo ello es que conducir bajo los efectos del alcohol es especialmente arriesgado para los jóvenes (ver Andréasson, 1991)

El número de infracciones de tráfico cometidas por jóvenes que han ingerido previamente alcohol y de las que la policía ha tenido conocimiento ha ido disminuyendo en Suecia desde mediados de los setenta. Sin embargo, esos datos no parecen reflejar con exactitud el número de infracciones realmente perpetradas (ver Persson, 1980). Puesto que un elevado porcentaje de infracciones jamás son denunciadas, el número de infracciones conocidas es sensible al menor cambio, por ejemplo, en los controles policiales. Así, cuando el número de conductores obligados a detenerse en un control se reduce sistemáticamente, el porcentaje de infracciones causadas por el consumo excesivo de alcohol registradas por la policía también disminuye, con independencia del número de infracciones cometidas.

Las últimas tendencias en el consumo de alcohol per capita entre los jóvenes suecos no harían sino contradecir la idea de que el descenso en las infracciones registradas refleja el número de infracciones cometidas. Varios estudios indican que el consumo de alcohol entre los jóvenes ha aumentado, al menos durante la primera mitad de los noventa (Leifman, 2000; Kühlhorn y colaboradores, 2000; FHI \& CAN, 1999; Blazeninova, 1995). Sin embargo, al no tratarse de unos datos necesariamente fiables, nos abstendremos de sacar unas conclusiones demasiado trascendentales. Todo ello nos lleva a preguntarnos si las tendencias en las infracciones debidas al exceso de alcohol registradas por la policía reflejan realmente las tendencias en el número de infracciones perpetradas.

$Y$ ésta es la pregunta que planteamos en nuestro artículo: ¿el descenso del número de infracciones relacionadas con el consumo excesivo de alcohol registradas significa que ha disminuido el número de jóvenes suecos que conducen después de haber ingerido alcohol?

Figura 1.- Número de infracciones de conductores que han ingerido alcohol conocidas por la policía cada 1000 habitantes (a partir de 15 años) entre 1975 y 1997. Índice $1975=100$ ( ${ }^{\circ}$ de infracciones registradas en $1975=21,685$ ).

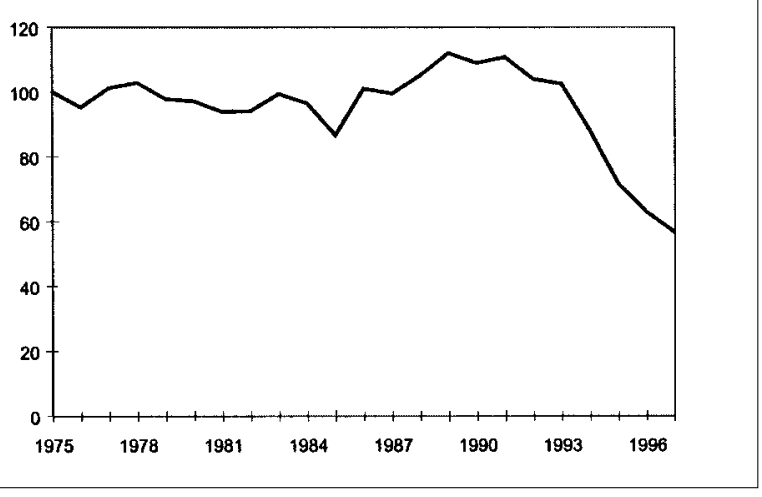

\section{Cambios experimentados en las infracciones debi- das al consumo excesivo de alcohol registradas por la policía.}

La figura 1 nos muestra el número de infracciones que han llegado a conocimiento de la policía entre 1975 y 1997. Los datos han sido extraídos de las estadísticas oficiales suecas. La ventaja principal de estudiar el número de las infracciones registradas es que las estadísticas se centran en las infracciones y no en el número de personas que se han visto implicadas: ello nos aporta una visión de las tendencias reales en las infracciones registradas, con independencia de las personas que se han visto envueltas. Tal como podemos observar, el número de infracciones conocidas por la policía fue relativamente constante entre 1975 y 1987. Es muy probable que el brusco descenso experimentado en 1985 se debiera a una reorganización de las operaciones policiales que tuvo como consecuencia la reducción del número de policías de tráfico (Knutsson, 1992). Las infracciones registradas parecieron aumentar a finales de los ochenta. A partir de 1991, su número ha ido disminuyendo de forma espectacular, pasando de las 26,100 infracciones registradas en 1991 a las 13,551 de 1997. En otras palabras, el número de infracciones de tráfico motivadas por el consumo excesivo de alcohol se ha reducido casi un 50\% durante la década de los noventa. 
Infracciones de tráfico cometidas por jóvenes que han ingerido demasiado alcohol.

Los datos de los supuestos infractores, al igual que los de las infracciones registradas, se basan en las estadísticas oficiales suecas; en este caso se han establecido diferentes grupos en función de sus edades. Estas estadísticas reflejan el número de personas detectadas, no el de infracciones conocidas por la policía (cada posible infractor aparece una sola vez). El riesgo de que dos series temporales estadísticas reflejen diferentes tendencias en desarrollo es mínimo, aunque se basen en distintas unidades estadísticas. Eso significa que durante el período estudiado esas personas aparecen por término medio no más de 1,14 veces al año como posibles infractores. Además, el número de veces que los sospechosos aparecen en las series tiende a disminuir con el tiempo.

Figura 2.- Número de conductores sospechosos de haber cometido una infracción bajo los efectos del alcohol entre jóvenes (de 15 a 17 años, de 18 a 20 y de 15 a 20) y adultos (más de 21 años) por cada 1000 habitantes -en sus respectivos grupos de edad- entre 1975 y 1997. Índice $1975=100\left(\mathrm{n}^{\circ}\right.$ en 1975 : $15-17=1654 ; 18-20=2630 ; 15-20=4284$; $21+=12033$ ).

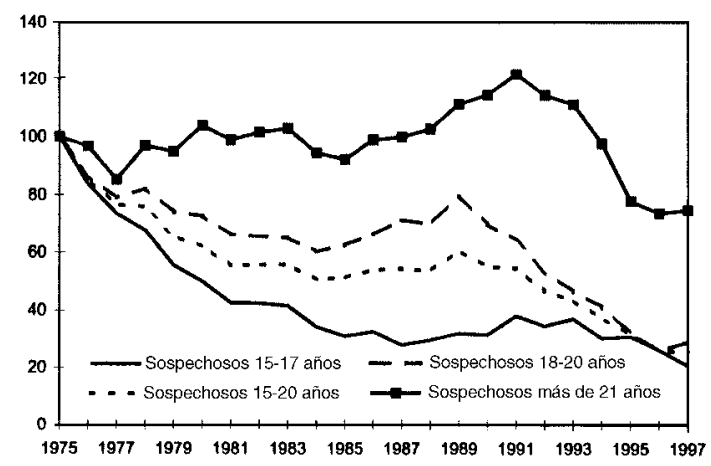

Tal como puede observarse en la figura 2, el número de infracciones cometidas por los jóvenes después de consumir alcohol ha disminuido claramente durante el período analizado. En el grupo de edad comprendido entre los 15 y los 20 años, el número de posibles infractores se reduce desde 1975 hasta principios de los ochenta. A partir de entonces, las cifras se mantienen bastante estables hasta finales de esa misma década. Desde 1990 hasta 1997, el porcentaje tiende a disminuir.

Existen algunas diferencias entre las pautas de conducta de los jóvenes y de los adultos. La tendencia general es similar hasta 1977, aunque las cifras de adultos se reducen menos bruscamente. En 1978, sin embargo, los dos grupos toman rumbos muy diferentes. En 1978, entre el grupo de más de 21 años, el número de posibles infractores vuelve a ser el mismo de 1975, y no experimenta la menor variación hasta mediados de los ochenta, cuando las cifras comienzan a incrementarse. El número de infracciones registradas empieza a disminuir claramente desde principios de los noventa.

Existe una lógica línea divisoria entre los jóvenes con edad suficiente para obtener el permiso de conducir y los que aún no han cumplido los dieciocho años. El número de posibles infractores entre los jóvenes de 15 a 17 años y los de 18 a 20 revela unas sorprendentes diferencias. Así, la figura 2 describe el número de posibles infractores entre los jóvenes y los menos jóvenes. Entre los primeros, el número disminuye a mediados de los ochenta y permanece estable hasta principios de los noventa, cuando las cifras caen ligeramente. Entre los segundos, el número de posibles infractores se reduce hasta mediados de los ochenta, cuando vuelve a aumentar hasta 1989. A partir de 1990 (con la única excepción de 1997), el número de infractores que han consumido demasiado alcohol entre los 18 y los 20 años desciende bruscamente.

\section{En busca de una interpretación.}

En Suecia, conducir bajo los efectos del alcohol es un delito que suele detectarse de cuatro modos diferentes:

-El infractor es identificado en un control de alcoholemia aleatorio en la carretera.

- La policía detiene un vehículo tras observar algo sospechoso en él.

-Se recibe la denuncia de otro ciudadano.

-El infractor se ve envuelto en un accidente de tráfico.

A grosso modo, la mitad de las infracciones relacionadas con el consumo excesivo de alcohol registradas por la policía están relacionadas con accidentes de tráfico y con una conducción sospechosa; un 25\% se detectan en los controles de alcoholemia y un $25 \%$ gracias a la colaboración de otros ciudadanos (BRA, 1998; JuU2, 1989-90; ver también Klette, 1970). Al igual que ocurre con otros delitos sin víctimas donde la detección está basada en la intervención activa de la policía, sólo algunas de las infracciones de tráfico motivadas por el consumo excesivo de alcohol llegan a ser conocidas. Ello dificulta enormemente el estudio de su evolución. El número latente de infracciones, o la relación entre el número de infracciones que se cree han sido cometidas y el número de infracciones registradas se desconoce por definición, pero es especialmente elevado en los delitos en los que no se produce ninguna víctima. 
En Suecia, se han realizado algunos intentos para determinar el número latente de infracciones de tráfico motivadas por el consumo de alcohol. Persson (1980) calcula que por cada una de las infracciones que llegan a conocimiento de la policía se cometen doscientas que pasan desapercibidas para ésta. En 1996, la Red de Carreteras Suecas y el Instituto del Transporte (VTI) se unieron a la Policía Nacional (RPS) en un ambicioso proyecto para evaluar el verdadero alcance del problema en el país. Las estimaciones preliminares basadas en su informe indican que en 1996 se cometieron más de cuatro millones de infracciones de tráfico después de ingerir demasiado alcohol, lo que significa que por cada infracción denunciada se producen cerca de trescientas de las que nadie da parte. Sin embargo, este último estudio no es directamente comparable con el de Persson. Con el fin de valorar la magnitud del cambio en la proporción, es necesario medir cuidadosamente, de forma idéntica y al menos en dos ocasiones diferentes, las infracciones de tráfico motivadas por el consumo excesivo de alcohol. Por ese motivo, no podemos ofrecer una explicación fidedigna a la variación experimentada por esa cifra. Lo que sí podemos afirmar con certeza es que el número de infracciones de tráfico bajo los efectos del alcohol es muy elevado, y que resulta difícil determinar hasta qué punto las infracciones registradas reflejan las infracciones realmente cometidas.

La cifra de infracciones perpetradas por cada una de las que llegan a conocimiento de la policía se ve influenciada principalmente por los efectos atribuibles al mismo instrumento de medida, es decir los cambios que socavan la comparabilidad de una medida determinada entre al menos dos puntos de medición, lo que significa, por ejemplo, que existen cambios sistemáticos en un indicador que no tiene correspondencia con las variaciones experimentadas por el delito real (Campbell \& Stanley, 1963). Un efecto atribuible al mismo instrumento de medida se produce cuando una parte del cuestionario se expresa de forma diferente entre dos mediciones. Aunque se dé por sentado que las preguntas evalúan el mismo fenómeno, un cambio en la redacción puede llevar a una valoración muy diferente, incluso cuando el resto del cuestionario permanece invariable. Por ese motivo, es de suma importancia tener en cuenta los efectos atribuibles al mismo instrumento de medida a la hora de interpretar los resultados empíricos.

\section{Una interpretación posible.}

El análisis se basa en dos hipótesis de trabajo. La primera de ellas se centra en los efectos atribuibles al mismo instrumento de medida. Habría que determinar en primer lugar si el descenso del número de infracciones de tráfico relacionadas con el consumo excesivo de alcohol puede atribuirse a distintos efectos atribuibles al mismo instrumento de medida.

Si no existe una clara evidencia de que esa disminución sea debida fundamentalmente a dichos efectos, es necesario pasar a nuestra segunda hipótesis de trabajo: el descenso del número de infracciones de tráfico relacionadas con el consumo excesivo de alcohol es debida básicamente al hecho de que se han cometido menos infracciones reales. Cuando las estadísticas se analizan a partir de esta hipótesis, es importante que la tendencia sea validada al menos por un criterio independiente.

En el presente estudio emplearemos datos conseguidos a través de encuentras autoadministradas sobre la conducta al volante de los jóvenes después de ingerir demasiado alcohol como un criterio independiente. Con unas muestras representativas del país, el Centro de Estadísticas Sueco ha realizado encuestas anuales para estudiar las actitudes de los individuos hacia la seguridad vial y su comportamiento en las carreteras desde 1981. El número de respuestas ha oscilado entre 2400 y 7200 . El porcentaje de respuestas se ha mantenido entre el $75 \%$ y el $80 \%{ }^{2}$. Las infracciones de tráfico debidas al consumo excesivo de alcohol se han evaluado basándose en la siguiente pregunta: En los últimos doce meses, ¿ha conducido en alguna ocasión después de beber alcohol (a excepción de cerveza baja en alcohol)?

Podría discutirse hasta qué punto esta pregunta mide el alcance de las infracciones cometidas bajo los efectos del alcohol. Dejando a un lado las habituales fuentes para evaluar el error, la formulación de la pregunta da pie a una serie de problemas de validez, pues esta definición operacional de conducir tras ingerir alcohol es más amplia que la definición nominal. Conducir un coche después de beber alcohol (a excepción de cerveza baja en alcohol) no es necesariamente un delito, ya que el conductor puede estar por debajo del mínimo tolerado (BAC 0,02\%). Sin embargo, esa medición tiene una importante ventaja: la pregunta ha sido repetida exactamente igual todos los años, lo que significa que el problema de validez no ha podido empeorar. Con todo, su empleo debe limitarse a calcular la tendencia a conducir después de haber consumido alcohol.

Para evaluar las tendencias en el número de infracciones registradas es necesario analizar el número de conductores entre 15 y 19 años que han resultado muertos en accidentes de tráfico, según las estadísticas policiales. Las estadísticas sobre el número de

\footnotetext{
${ }^{2}$ Las encuestas habían sido encargadas por el Centro de Seguridad Vial y, más recientemente, por la Administración de las Carreteras Nacionales. El servicio informativo del Instituto de Ciencias Sociales Suecas me facilitó el material para la investigación. Sin embargo, los análisis y las interpretaciones del presente estudio son únicamente míos.
} 
conductores fallecidos en las carreteras se basan en los accidentes mortales notificados a la policía. Son publicadas por el Centro Estadístico Sueco y están a disposición de quien desee consultarlas hasta el año 1997. Puesto que no nos permiten diferenciar entre el número de conductores y el de pasajeros, quedan excluidas de nuestro análisis. Éste debe centrarse en los conductores de coches con o sin acompañantes, así como en los de motocicletas. Existen razones que nos hacen suponer que ello ha supuesto un enorme impacto en la interpretación de los resultados para los más jóvenes (15-17 años).

Tal como hemos señalado antes, los conductores que han ingerido alcohol corren un riesgo considerablemente mayor de verse envueltos en accidentes mortales. Aunque se estima que no hay más de dos conductores en ese estado por milla en el conjunto del tráfico (ver Persson, 1980), se ha demostrado que casi el $25 \%$ de los conductores que se ven envueltos en accidentes mortales están bajo los efectos del alcohol (Östrom \& Eriksson \& Björnstig, 1993). Sin embargo, tampoco deja de ser un problema utilizar el número de conductores muertos como un indicativo del aumento de las infracciones motivadas por el consumo excesivo de alcohol. Está claro que no existe una asociación directa entre el número de conductores que han bebido demasiado alcohol registrados y el de conductores muertos. Tanto el número de posibles infractores como del de conductores fallecidos dependen de una serie de factores muy diferentes. No obstante, si asumimos que existe una cifra constante de infracciones sin registrar por cada una de las que sí llegan a conocimiento de la policía, una elevada proporción significa que incluso el menor cambio en el número de infracciones registradas supone un cambio significativamente mayor en el número de infracciones realmente perpetradas. Por ejemplo, si el número de infracciones derivadas del consumo de alcohol que llega a conocimiento de la policía desciende en 100, ello significaría (asumiendo que por cada 1 denunciada se produzcan verdaderamente 200) que se han cometido 20000 infracciones menos. Dado el enorme riesgo de verse envueltos en un accidente mortal que corren los conductores que han ingerido alcohol, el número de conductores muertos debería seguir por extensión la misma tendencia que el número de infracciones registradas, asumiendo que las infracciones reales son más o menos constantes.

Sin embargo, existen algunos datos que nos llevan a pensar que el número de infracciones cometidas por cada una registrada podría haber variado durante el período analizado; una serie de cambios en la utilización de los recursos y en las prácticas policiales apuntan en esa dirección. Cualquier variación repentina en la cifra de infracciones verdaderamente cometidas conduciría a la interpretación equivocada de los datos de las infracciones registradas. El criterio de validez es, por consiguiente, utilizado para controlar los cambios demasiado bruscos en la cifra de las infracciones que no llegan a conocimiento de la policía. Si las distintas series señalan tendencias muy diferentes con el paso del tiempo, existen razones para dudar que las variaciones en el número de infracciones registradas reflejen un verdadero cambio.

\section{¿Refleja el descenso una verdadera disminución en el número de infracciones?}

Nuestra primera hipótesis parte de la base de que la disminución en el número de posibles infractores jóvenes puede explicarse haciendo referencia a varios efectos atribuibles al mismo instrumento de medida. Esta hipótesis deberá ser rechazada a menos que esos efectos lógicos sean consecuentes con los datos empíricos.

\section{Estadísticas.}

Durante el período estudiado, las estadísticas oficiales sobre los posibles infractores ${ }^{3}$ han experimentado algunos cambios. Antes de 1987, la edad se determinaba en función de la edad a la que se había cometido la primera infracción; en 1986, esta definición fue modificada y pasó a ser la edad cumplida por el infractor durante el año en que se le abre por primera vez un expediente.

Esa revisión tiene importantes implicaciones en relación a la comparabilidad en el tiempo. Adoptando esa nueva definición de edad, las estadísticas muestran un número sistemáticamente menor de posibles infractores jóvenes (ver Knutsson -1997- para un análisis en profundidad de este cambio y sus efectos). El problema ha sido obviado empleando únicamente los datos donde se había aplicado la anterior definición de edad.

El descenso del número de infracciones bajo los efectos del alcohol entre los jóvenes no puede, por tanto, deberse a la adopción en 1987 de una nueva definición de edad en las estadísticas de los posibles infractores. Además, el número de jóvenes infractores comenzó a disminuir mucho antes de 1987.

\section{Cambios en la legislación.}

Los cambios en la legislación guardan, asimismo, relación con la exactitud con que las infracciones

\footnotetext{
${ }^{3}$ Las estadísticas de los posibles infractores se basan en las fichas personales recopiladas por la policía en aquellos casos en los que existen motivos razonables para sospechar. Los jóvenes sin edad suficiente para ser castigados se han excluido de las estadísticas.
} 
registradas reflejan las tendencias reales en desarroIlo. Durante el período en estudio (1975-1997) se produjeron varios cambios en la legislación que afectaron a las infracciones de tráfico motivadas por el consumo excesivo de alcohol. Uno de esos cambios repercutió especialmente en la comparabilidad de las estadísticas sobre esa clase de infracciones a lo largo del tiempo. El 1 de julio de 1990, la concentración legal de alcohol en la sangre fue reducido de 0,05 a 0,02\%. Se ha estimado que el número de infracciones después de ingerir alcohol aumentó un 13\% como consecuencia directa del nuevo límite permitido ${ }^{4}$.

Si damos por supuesto que la policía no ha realizado cambios operativos y que el número de infracciones reales por cada una registrada se mantiene constante, el aumento de las infracciones punibles llevaría a detectar un número mayor de conductores que han consumido excesivo alcohol. Aunque las operaciones policiales no han sido constantes durante el período estudiado, esa clase de cambios en la legislación, dado que se necesita algún tiempo para introducirlos en la conciencia pública, parecen destinados a incrementar al menos temporalmente el número de infracciones cometidas.

Es difícil saber con exactitud qué pudo haber hecho la policía, no sólo para impedir el crecimiento, sino también para disminuir las infracciones protagonizadas por conductores entre 18 y 20 años que habí- an consumido demasiado alcohol, mientras que las cifras se incrementaban ligeramente entre los más jóvenes. La única prueba de un efecto legislativo es el aumento temporal del número de jóvenes infractores entre 1990 y 1991, pero aquella circunstancia fue seguida de un descenso tan sólo un año después. El límite más bajo de nivel de alcohol en sangre no condujo a un incremento en el número de infracciones registradas entre los jóvenes de 15 a 20 años. Eso significa que el descenso en el número de posibles infractores entre 15 y 20 años habría sido más brusco de no haber entrado en vigor la nueva ley.

\section{Controles policiales.}

Los cambios en las prácticas judiciales pueden influir en el número total de infracciones registradas. Dada la naturaleza de las infracciones de tráfico derivadas del consumo excesivo de alcohol, cualquier endurecimiento de las prácticas policiales debería aumentar inmediatamente el riesgo de ser descubierto $y$, del mismo modo, el número de infracciones registradas.

Durante la década de los noventa, la policía adopta una serie de nuevas estrategias para endurecer la lucha contra quienes conducen bajo los efectos del alcohol. También experimenta ciertos cambios en su poder. A partir de 1994, cualquier oficial de policía

Tabla 1.- $N^{\circ}$ de infracciones cometidas bajo los efectos del alcohol registradas por la policía, $n^{\circ}$ de tests de capacidades realizados, $\mathrm{n}^{\circ}$ estimado de casos detectados en controles rutinarios, $\mathrm{n}^{\circ}$ estimado de infracciones detectadas al margen de dichos controles: 1987, 1991,1994 y 1997.

\begin{tabular}{|lcccc|}
\hline Año & $\begin{array}{c}\text { infracciones } \\
\text { cometidas bajo } \\
\text { los efectos del alcohol }\end{array}$ & $\begin{array}{c}\mathbf{N}^{\circ} \text { de tests } \\
\text { de capacidades } \\
\text { realizados }\end{array}$ & $\begin{array}{c}\mathbf{N}^{\circ} \text { estimado de } \\
\text { casos detectados } \\
\text { en controles } \\
\text { rutinarios }\end{array}$ & $\begin{array}{c}\mathbf{N}^{\circ} \text { estimado de } \\
\text { infracciones detectadas } \\
\text { al margen de dichos } \\
\text { controles }\end{array}$ \\
\hline 1987 & 22923 & 683551 & 4768 & 18155 \\
1991 & 26100 & 930826 & 7517 & 18583 \\
1994 & 21011 & 1775877 & 6277 & 14734 \\
1997 & 13551 & 1145856 & 3493 & 10058 \\
$(\%)$ & & & & \\
$1987-1991$ & $+13,9$ & $+36,2$ & $+57,6$ & $-2,4$ \\
$1991-1994$ & $-19,5$ & $+90,8$ & $-16,5$ & $-31,7$ \\
$1994-1997$ & $-35,5$ & $-35,5$ & $-44,3$ & $-45,9$ \\
$1991-1997$ & $-48,1$ & $+23,1$ & $-53,5$ & \\
\hline
\end{tabular}

\footnotetext{
${ }^{4}$ Un examen de los datos obtenidos en los tests de alcoholemia en el Laboratorio Forense Nacional Sueco nos permite evaluar los efectos del cambio de ley. Para este cálculo se da por supuesto que la bajada del límite legal no tiene el menor efecto preventivo. Entre las personas que en 1994 pasaron el test por existir una sospecha razonable de que habían bebido en exceso, el 18\% tenían una concentración de alcohol en la sangre de menos de 0,05\%. En este grupo, casi el 40\% tenían menos del 0,02\% (Solarz, 1989). El 60\% restante, con una concentración de alcohol en la sangre entre el $0,02 \%$ y el 0,049\% corresponden al 13\% del $82 \%$ que tenían una concentración del 0,05\% o más. Esas cifras parecen coincidir con los datos presentados en el informe del comité encargado de este problema, según el cual el porcentaje correspondiente puede estimarse alrededor del 15\% (SOU, 1992:131).
} 
puede realizar tests rutinarios sin necesidad de pedir autorización a sus mandos 5 . El número de controles rutinarios ha pasado de 772.000 en 1989 a 1.776 .000 en 1994.

En los últimos años, sin embargo, ha disminuido el control de los motoristas. En 1997, se efectuaron aproximadamente 1.146.000 tests. Durante la década de los noventa, la policía cambia su política en relación con los tests aleatorios de alcoholemia: mientras que previamente su estrategia era concentrar esfuerzos en algunas horas, días y lugares, sus controles pasan a ser mucho menos predecibles.

A pesar de todas esas modificaciones en la estrategia policial, no existen razones para creer que el riesgo de ser descubierto haya aumentado tanto como para ser la única explicación al descenso de las infracciones registradas. Los cambios de procedimiento ideados por la policía no afectan al relativamente elevado número de infracciones motivadas por el consumo excesivo de alcohol que se detectan al margen de los controles rutinarios, de igual modo que a las infracciones descubiertas en éstos.

Si damos por sentado que el número de infracciones cometidas es constante y que la policía (antes de revisar su estrategia) detectó un $25 \%$ de los infractores en controles rutinarios, la policía debería haber dejado virtualmente de detectar infractores bajo los efectos del alcohol en los controles rutinarios con el fin de que el descenso en el número de infracciones pudiera justificarse por el menor riesgo de ser descubierto. No obstante, el número de infracciones detectadas mediante controles rutinarios, como un porcentaje de las infracciones detectadas de otro modo, ha sido relativamente estable durante la década de los noventa (BRA, 1998). Eso significa que el número de infracciones detectadas de otro modo, menos sensibles a los cambios de estrategia en los controles policiales, disminuyó en los años noventa.

Mientras la nueva estrategia policial redujo el porcentaje de infracciones detectadas en controles aleatorios (BRA, 1998), los conductores que habían ingerido demasiado alcohol continuaron siendo descubiertos en los controles rutinarios. La tabla 1 muestra que el número de infracciones motivadas por el consumo de dicha sustancia -detectadas al margen de los controles rutinarios- descendió muy bruscamente entre 1991 y 1994, y casi de igual modo entre 1994 y 1997, si lo comparamos con el número de infracciones detectadas en dichos controles (para una descripción de los procedimientos de evaluación ver BRA, 1998). El descenso observado a principios de los noventa se debe, por consiguiente, a la combinación de unos controles rutinarios menos efectivos y a un número menor de casos de conductores bajo los efectos del alcohol.

Las tendencias en desarrollo entre los diferentes grupos de edades (ver figura 2) también chocan con la idea de que el descenso del número de posibles infractores podría justificarse haciendo referencia al menor riesgo de ser descubierto. Es difícil percibir hasta qué punto cualquier cambio en los recursos puestos a disposición de la policía puede afectar de uno u otro modo a los jóvenes o a los menos jóvenes. Además, las pautas empiezan claramente a cambiar unos diez años antes del programa de fuertes inversiones puesto en marcha a finales de los ochenta-principios de los noventa para reforzar el control policial. La figura 2 indica que la edad de inicio de las infracciones derivadas del consumo de alcohol ha aumentado desde 1975, no que los recursos y las prácticas policiales hayan cambiado durante los últimos años del estudio.

\section{Claro descenso de las infracciones motivadas por el consumo excesivo de alcohol entre los jóvenes.}

Un análisis de los datos conseguidos a través de encuestas autoadministradas con el fin de medir la existencia de conductores bajo los efectos del alcohol en una muestra aleatoria de población normal confirma que el número de infracciones derivadas del consumo excesivo de alcohol ha descendido entre los jóvenes. La figura 3 describe, asimismo, las tendencias en el número de posibles infractores entre 15 y 20 años.

Los datos del Informe para la Seguridad Vial indican que la proporción de jóvenes que manifiestan haber conducido un coche después de ingerir alcohol (a excepción de cerveza baja en alcohol) ha disminuido desde finales de los ochenta. Esto sólo puede aplicarse a aquellos encuestados que afirman haber conducido un coche en los últimos doce meses. En esas series temporales, las tendencias reflejan la existencia de infracciones bajo los efectos del alcohol cuando tenemos en cuenta el cambio del porcentaje de jóvenes que realmente conducen.

\footnotetext{
${ }^{5}$ Los niveles de concentración de alcohol en la sangre se determinan en los tests de alcoholemia o en los análisis de sangre. Sin embargo, la policía sólo tiene derecho a realizar esas pruebas cuando existen motivos razonables para sospechar que el conductor ha sobrepasado el límite. Ésa es la razón por la que el posible infractor deberá pasar un control rutinario de capacidades que ayudará a la policía a decidir si el nivel de alcohol excede o no un cierto límite. Si el resultado es positivo, el posible infractor deberá pasar el test de alcoholemia que será utilizado como una prueba en su contra. En aquellos casos en los que el sospechoso se niegue o sea incapaz de pasar el test, se le realizará un análisis de sangre.
} 
Figura 3.- Número de encuestados entre 15 y 20 años que aseguran haber conducido después de consumir alcohol (a excepción de cerveza baja en alcohol) y número de posibles infractores entre jóvenes de 15 a 20 años por cada 1000 habitantes. Índice 1981=100 (número de posibles infractores en $1981=2.597$, porcentaje según los datos del estudio, $1981=8.62 \%$ ).

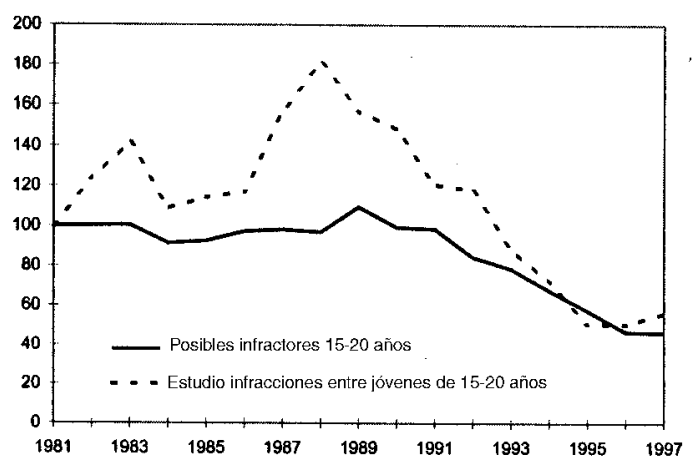

Sin embargo, se desconoce hasta qué punto ese descenso hace referencia tanto a una conducta legal como ilegal. No siempre que se conduce un coche después de ingerir alcohol se está cometiendo un delito; el nivel de alcohol en sangre puede estar por debajo del límite establecido. Norström y Andersson (1996) señalaron que la reforma de la ley en 1990 ocasionó un descenso global de los niveles de alcohol en sangre. Partiendo de esa base, no sería improbable que el descenso observado en los datos conseguidos a través de encuestas autoadministradas reflejara un descenso general en la tendencia a conducir después de consumir alcohol. Sin embargo, la causa de las bruscas variaciones experimentadas en la década de los ochenta no está suficientemente clara.

El segundo criterio empleado para evaluar las tendencias en el número de infracciones registradas es el porcentaje de víctimas de accidentes mortales. El número de conductores muertos entre 15 y 19 años (según las estadísticas de los accidentes de tráfico denunciados a la policía) es, en parte, una consecuencia directa de conducir bajo los efectos del alcohol. Si esto es así, sería lógico pensar en una asociación entre el número de conductores bajo los efectos del alcohol y el número de individuos que se ven envueltos en accidentes.

El número de conductores jóvenes muertos refleja una tendencia similar a la del número de infracciones registradas. Entre 1975 y 1997, el número total de conductores de más de catorce años que perdieron la vida disminuyó de 402 a 305. El mayor número de víctimas -411- fue registrado en 1989. Durante ese mismo período, el número de conductores fallecidos entre 15 y 19 años descendió de 57 a 6 . El número más elevado de víctimas mortales fue registrado en
1978 (63 jóvenes). La figura 4 muestra el número de conductores jóvenes sospechosos de haber cometido una infracción después de consumir alcohol y el número de conductores muertos.

Figura 4.- $\mathrm{N}^{0}$ de posibles infractores y $\mathrm{n}^{\circ}$ de conductores víctimas mortales de un accidente (15-19 años) por cada 1000 habitantes en los respectivos grupos de edad entre 1975 y 1997. Índice $1975=100\left(n^{\circ}\right.$ de posibles infractores $1975=3.454$; $\mathrm{n}^{\circ}$ de conductores muertos $1975=57$ ).

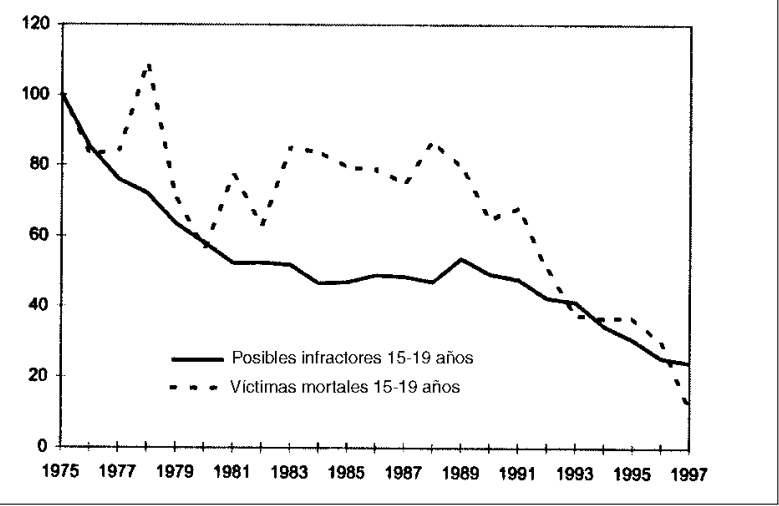

El porcentaje de jóvenes de más de 15 años víctimas de algún accidente de tráfico desciende claramente desde 1983 hasta 1993; a partir de entonces se mantiene constante en relación a los accidentes mortales en el grupo de edad de 14 o más años. Durante la primera parte del período estudiado, el $14 \%$ de los conductores que perdían la vida en la carretera tenían entre 15 y 19 años. Al final, el porcentaje correspondiente era sólo de un $6 \%$, si exceptuamos 1997, año en el que la cifra se redujo al $2 \%$. El hecho de que los accidentes de tráfico mortales sean menos frecuentes entre los jóvenes, tanto en términos absolutos como relativos, parece probar la existencia de un descenso real de infracciones motivadas por el consumo excesivo de alcohol.

Una explicación posible (y lógica) del descenso de los accidentes mortales y de las infracciones bajo los efectos del alcohol es el número cada vez menor de conductores jóvenes en las carreteras. Un estudio reciente indica que la mera presencia de jóvenes conductores tiene un claro efecto en el número total de accidentes mortales (Brüde 1999). Para determinar si ésta puede ser la única explicación del descenso en el número de infracciones registradas y en el número de accidentes mortales, podemos estandarizar los datos de las infracciones y de los accidentes mortales por el número de conductores o por el número total de kilómetros recorridos. Existen dos poderosas razones más por las que el número de posibles infractores y de conductores muertos deberían relacionarse con los kilómetros conducidos más que con el número de conductores potenciales, es decir individuos con 
carné de conducir. En primer lugar, un elevado porcentaje de conductores en general y de jóvenes en particular que han bebido alcohol no tienen carné de conducir y, en segundo lugar, parece que un descenso en la proporción de jóvenes que tienen carné queda compensado por un aumento en la proporción de infractores que conducen sin él (BRA, 1998).

El número de conductores víctimas de un accidente mortal y el número de posibles infractores guardan relación en cambio con el número de jóvenes que se ven inmersos en el tráfico de las carreteras. Con el fin de evaluar esa cuestión, nos basamos en el número de kilómetros conducidos por los jóvenes al año. Obtenemos esos datos del Informe para la Seguridad Vial y abarcan el período comprendido entre 1981 y 1997. Puesto que sólo los coches tienen kilometraje, el número de posibles infractores y de víctimas mortales por cada 100 millones de kilómetros está ligeramente sobreestimado. Sin embargo, es algo que debería carecer de importancia, ya que el kilometraje total de las motocicletas es mínimo en comparación con el de los coches.

Figura 5.- Número de posibles infractores y de conductores muertos (entre 15 y 19 años), por cada 100 millones de kilómetros, desde 1981 hasta 1997. Índice $1981=100$ ( $n^{\circ}$ de posibles infractores $1981=2.016, \mathrm{n}^{\circ}$ de conductores muertos $1981=49)$.

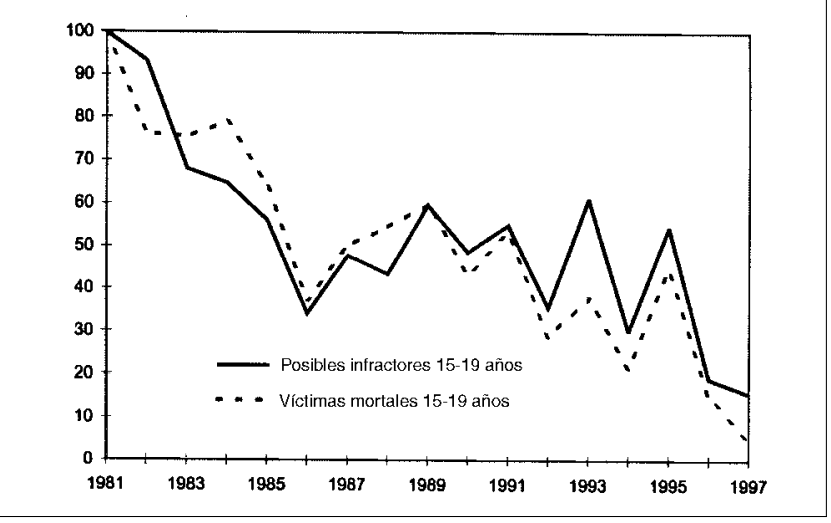

La figura 5 muestra cuáles son las tendencias en el número de conductores fallecidos y en el número de posibles infractores entre 15 y 19 años, por cada 100 millones de kilómetros recorridos por vehículos. Tal como podemos observar, el número de infracciones derivadas del consumo excesivo de alcohol que han sido registradas y de conductores fallecidos entre los 15 y los 19 años ha descendido incluso teniendo en cuenta que conducen menos jóvenes que antes. La mayor correspondencia entre las series temporales de la figura 5, en comparación con las de la figura 4, puede explicarse por los diferentes períodos de tiempo comprendidos.

\section{Discusión.}

El presente estudio ha mostrado que el descenso observado en el número de infracciones cometidas en Suecia por jóvenes que conducen bajo los efectos del alcohol no puede explicarse de manera convincente con los efectos atribuibles al mismo instrumento de medida: las tres razones clave que hemos analizado en este sentido no han logrado proporcionar una explicación adecuada a esa disminución. En primer lugar, hemos estudiado las estadísticas de los posibles infractores. El análisis, asimismo, ha tenido en cuenta los distintos grupos de edades, algo de enorme importancia en los estudios de delincuencia juvenil (ver Knutsson, 1997). En segundo lugar, la reforma legislativa, que cualquier análisis teórico habría creído de enorme influencia sobre el número de infracciones registradas, no ha tenido más que una influencia marginal en las tendencias de las infracciones de los jóvenes conductores después de beber alcohol. La disminución del límite legal de nivel de alcohol en sangre -que ha pasado del 0,05\% al 0,02\%-no parece haber incrementado el número de infractores, sino haber retardado el descenso. Por último, no parece probable que los cambios en la política de vigilancia puedan justificar la disminución del número de infracciones. Si ésta fuera debida exclusivamente al menor número de casos detectados en los controles policiales, no observaríamos un descenso tan brusco en el número de infracciones detectadas por otros métodos al margen de los controles rutinarios: accidentes de coche, denuncias de otros ciudadanos, conducción sospechosa... Desde 1991 hasta 1997, el número de infracciones bajo los efectos del alcohol que han sido detectadas de alguno de esos modos ha disminuido de forma muy similar.

Estudios anteriores han mostrado cómo la intensificación de los controles policiales influye también en la conducta de los individuos, al reducir los niveles de actividad (ver Homel, 1990). Una explicación a lo sucedido a principios de los noventa podría ser que el riesgo objetivo y, por tanto, subjetivo de ser descubierto ha aumentado debido al mayor número de controles rutinarios, unido al hecho de que la policía tiene mayores poderes y herramientas más sofisticadas (Aberg, 1986). Un número más elevado de controles puede tener como resultado, en otras palabras, un número menor de infracciones, incluso cuando el porcentaje de casos detectados en los controles se haya reducido. Un riesgo más elevado de ser descubierto, por consiguiente, no sólo lleva a una nueva distribución de las infracciones, sino también a reducir los niveles de actividad.

Asimismo, nuestro estudio puso en evidencia que el descenso no podía justificarse con los cambios estructurales. Las dos explicaciones estructurales más plausibles de esa reducción en el número de infrac- 
ciones bajo los efectos del alcohol serían las cifras cada vez menores de consumo per capita entre los jóvenes y la menor presencia de éstos en las carreteras. Tal como señalamos antes, no parece que el consumo de alcohol entre los jóvenes haya disminuido en Suecia. Tampoco parece probable que el porcentaje menor de infracciones sea debido a un cambio en las pautas de consumo de alcohol entre los jóvenes. Por ejemplo, no existe la menor evidencia de que beber hasta emborracharse sea menos frecuente que antes (FHI \& CAN, 1999).

El número de conductores muertos que, según la autopsia, tenían alcohol en la sangre disminuyó a finales de los ochenta-primera mitad de los noventa (ver Leifman, 1995; Laurell, 1994). A pesar de las más bien bruscas fluctuaciones en las series temporales de conductores fallecidos (en accidentes de tráfico denunciados) entre los jóvenes de 15 a 19 años, las tendencias son bastante similares a las de las infracciones bajo los efectos del alcohol registradas por la policía. Estas pautas vuelven a repetirse cuando el número de víctimas mortales y de posibles infractores se relacionan con el número de kilómetros recorridos por los coches. En otras palabras, la presencia menor de los jóvenes en las carreteras tampoco explica el descenso. Además, el número de jóvenes que aseguran haber conducido un coche después de beber alcohol se ha reducido en la década de los noventa. El análisis tiene en cuenta si el encuestado ha conducido realmente un coche en los últimos doce meses.

Los resultados del presente estudio indican que ha existido un verdadero descenso en las cifras de infracciones motivadas por el consumo de alcohol entre los jóvenes suecos, especialmente durante los años noventa. La explicación más plausible de este fenómeno residiría en la aplicación regular en el país de una estrategia preventiva de éxito, a menudo descrita como el modelo escandinavo. Nuestra legislación actual para combatir el problema de los individuos que conducen bajo los efectos del alcohol es el resultado de un largo proceso de evolución que se remonta a 1923. A lo largo de los años y de las décadas, la ley se ha hecho cada vez más restrictiva y el control se ha endurecido. Sin duda, eso no habría sido posible sin el apoyo y el respaldo de los ciudadanos: Ios cambios tienen que ser consecuentes con el sentido de la justicia (ver Leifman, 1992; Lindén \& Similä, 1982; Axberger, 1996; Törnros, 1995).

La actual legislación sueca es, en muchos aspectos, única. El límite tolerado de alcohol es muy bajo, las multas por las infracciones muy severas y la policía tiene amplios poderes. Los límites fijados y la disponibilidad de los tests de capacidades han servido para incrementar la predictibilidad. Los infractores saben que si son descubiertos serán acusados de un delito criminal, lo que es una parte importante de la hipótesis de prevención general. Desde principios de los noventa, los resultados de los tests de alcoholemia han servido para probar con toda legitimidad el exceso de alcohol. Ello significa que los infractores no tienen tiempo de recuperarse mientras la policía llama al hospital para que vengan a realizar un análisis de sangre.

Las reformas destinadas a evitar que los individuos conduzcan bajo los efectos del alcohol introducidas en los años noventa han contribuido a reducir el número de infracciones registradas (BRA, 1998; Norström \& Andersson, 1996). Otra explicación posible sería el cambio de actitud general hacia ese problema; ello podría atribuirse a las campañas informativas de los noventa y a la difusión dada por los medios de comunicación, que han insistido no sólo en la nueva legislación, sino también en el problema social que supone conducir después de haber ingerido alcohol. El descenso del número de infracciones de esas características entre los jóvenes podría deberse a la estrategia preventiva, que ha puesto en marcha diferentes medidas, como por ejemplo el endurecimiento de las leyes, un mayor control policial y campañas informativas.

Para terminar, los resultados del presente estudio son sumamente interesantes desde el punto de vista de una política contra el alcohol, tanto por los cambios realizados en la estrategia de prevención como por los cambios que podemos esperar ver en el panorama sueco del alcohol. Basándose en el llamado modelo de consumo total (ver Olsson, 1990), la principal estrategia preventiva en Suecia se ha centrado desde hace mucho tiempo en intentar reducir el consumo total y, de ese modo, los daños del alcohol en la sociedad. Así, pues, durante las pasadas décadas el objetivo prioritario de la política sueca contra el alcohol ha sido restringir su disponibilidad y demanda.

La integración de Suecia en la Unión Europea ha tenido importantes consecuencias en la política de este país contra el alcohol (ver Trolldal, 1998). Convertirse en un miembro más de la Unión Europea ha hecho más difícil para Suecia mantener su política tradicional contra el alcohol, pero el objetivo final sigue siendo el mismo: reducir los efectos nocivos del alcohol (Dir. 1997:3). Sin embargo, las herramientas tradicionales de su política tendrán probablemente que dejar paso a nuevos métodos y estrategias (SOU, 1998:154).

Conducir bajo los efectos del alcohol ha sido considerado desde hace mucho tiempo uno de los mayores problemas relacionados con el consumo de esta sustancia, y algo contra lo que hay que luchar. El presente estudio ha mostrado que es posible, con la ayuda de la legislación y de las campañas informativas, reducir algunos de los problemas relacionados con el alcohol sin necesidad de introducir cambios en el consumo medio de alcohol o en las pautas de consumo. Eso es algo que no debemos olvidar mientras el país continúa trabajando para formular una política 
contra el alcohol en un entorno cada vez más internacional.

\section{Bibliografía.}

Andréasson, R. (|99|): Läkemedel, narkotika och körkort. Stockholm: Fischer \& Co

Andréasson, R., \& Jones, W. (1999): Alkohol och trafikbrott. En uppgift för rättskemin. Rättsmedicinalverket. RMV-rapport 1999:2. Stockholm: Fritzes

Axberger, H-G. (1996): "Det allmänna rättsmedvetandet". Brottsforebyggande rådet, BRÅrapport 1996:1. Stockholm: Fritzes

Blazeninova, K. (1995) : Svenska ungdomar och alkohol. En beskrivande empirisk studie av ungdomars alkoholkonsumtion i början på 90-talet med en diskussion kring möjligheten att tillämpa teorier om alkoholvaneutveckling i ung ålder i undersökning med tvärsnitt- respektive longitudinell design. Manuskript. Stockholm: Sociologiska Institutionnen. Stockholms universitet

BRÅ (IBrottsförebyggande råclet) (1998): Rattfylleri. Utvärdering av 1994 års reform av trafiknykterhetslagstiftnigen. BRÅ-rapport 1998:7. Stockholm: Fritzes

Brüde, U. (1999): Utvecklingen för antal trafikdodade. Hänsyn tagen till förändringar i antal unga bilförare samt alkohol i trafiken. KFB \& VTI forskning 28. Linkoping: Statens Väg- och transportforskningsinstitut

Campbell, D.T. \& Stanley, J.C. (1963): Experimental and Quasi-Experimental Designs for Research. Chicago: Rand McNally College Publishing Company

Direktiv 1997:3. Samverkan angående information kring bruk av alkohol, dess risker och skadeverkningar mellan branschorgainisationer, försäkringsbolag och berörda myndigheter

FHI \& CAN (Folkhälsoinstituter och Centralförbundet för Alkohol- och Narkotikaupplysning) (1999): Alkohol- och narkotikautvecklingen i Sverige, rapport 99, nr 8 Stockholm Ljunglöfs

Goldberg,L (1970): Alcohol och trafikrister. I: SOU 1970:61 Trafiknykterhetsbrott. Betänkande avgivet av kommittén för lagstiftning angående trafiknykterhetsbrott. Stockholm: Justitiedepartementet

Gregersen, N.P. (1996) : Young drivers' overestimation of their own skill. An experiment on the relation between training strategy and skill. Accident analysis and prevention (2): 243-250
Gregersen, N.P. (1997): Unga förare en högriskgrupp i trafiken. Epidemiologi, experimentella studier och förslag till analysmodell. Socialmedicinsk Tidskrift (2-3): 85-91

Homel, R. (1990) : Random breath testing and random stopping program in Australia. In: Wilson, J.R. BC Mann, R.E. (eds.) : Drinking and driving. Advances in research and prevention. New York: The Guilford Press

Justitieutskottet 1989/90 JuU2. Trafiknykterhetsbrotten m.m.

Kendell, R.E. (1984): The beneficial consequences of the United Kingdom's declining per capita consumption of alcohol in 1979-82. Alcohol \& Alcoholism 19: 27l-276

Klette, H. (1970): Orn upptäckten av trafiknykterhetsbrott. In: SOU 1970:61 Trafiknykterhetsbrott. Betänkande avgivet av kommittén för lagstiftning angående trafiknykterhetsbrott. Stockholm: Justitiedepartementet

Knutsson, J. (1992): Ökad eller stabit straffnivå. RPS Forskning 1992:3. Stockholm: Polishögskolan, forskningsenheten

Knutsson, J. (1997): Minskad eller ökad ungdomsbrottslighet - en studie i konsten att tolka kriminalstatistik. Stencil. Stockholm: Brottsförebyggande rådet

Kühlhorn, E. \& Hibell, B. \& Larsson, S. \& Rarnstedt, Pvt. \& Zetterberg, H.L. (2000): Alkoholkonsumtionen i Sverige under 1990-talet. Preliminart manuskript i stencil. Stockholm: Sociologiska institutionen, Stockholms universitet

Laurell, H. (1994) : Promillen och trafiksäkerheten. En kunskapsöversikt om alkohol och trafik. Forskning och fakta, nr 13, Stockholm: CAN \& SAD

Leifman, H. (I 992) : Om de senasre ändringarna i trafiknykterhetslagstiftningen. Manuskript, Stockholm: Sociologiska institutionen, Stockholms universitet

Leifrnan, H. (1995) : Alcohol and primary prevention in Scotland and Sweden: A cornparative study. Nordisk Alkoholtidskrift 12 (English Supplement): 3|-60

Leifman, H. (2000): Ungdomar och alkohol: trender och individuell utveckling - en genomgång av litteratur på området. Stockholm: OAS, Social departementet

Lindén, P-A. \& Similä, M. (1982): Rättsmedvetandet i Sverige. Rapport 1982:1. Stockholm: Brottsförebyggande rådet

Norström, T. R Andersson, J. (1996): Utvärdering av 1990 års reform av trafikbrottslagen. Manusunderlag av Thor Norstrom och Jan Andersson. Manuskript. Stockholm: Brottsförebyggande rådet 
Olsson, O. (1990): Totalkonsumtionsmodellen. En forskningsöversikt. CAN-rapport nr 12. Stockholm: Centralforbundet för alkohol- och narkotikaupplysning

Persson, L. (1980) : Hidden criminality - theoretical and methodological problems, empirical results. Dissertation, Stockholm: Department of Sociology, Stockholm Universiry

Ross, H.L. (1982): Deterring the drinking driver. Legal policy and social control. Toronto: Lexington books

Smart, R.G. \& Mann, R.E. (1987): Large decrease in alcohol-related problems following a slight reduction in alcohol consumption in Ontario 197583. British Journal of Addiction 82: 285-291

Solarz, A. (1989): Behövs en promillesänkning? In: VTImeddelande 590. Linköping: Statens vägoch trafikinstitut

SOU 1992:131 Grovt rattfylleri och sjöfylleri. Betänkande av Väg- och sjöfyllerikommittén. Stockholm: Allmänna Förlaget

SOU 1998:154 OAS i framtiden. Betänkande av Kommittén om samverkan angående information kring bruk av alkohol, dess risker och skadeverkningar mellan branschorganisationer, försäkringsbolag och berörda myndigheter OAS). Stockholm: Socialdepartementet

Spolander, K. (1992): Män och kvinnor bakomratten: nya data om deras olycksrisker - likheter och olikheter, orsaker och trafiksäkerhetsatgärder. Stockholm: Statistiska centralbyrån

Trolldal, B. (1998): Ändrade villkor för svensk alkoholpolitik. In: Kühlhorn, E. \& Björ, J. (red.): Svenska alkoholvanor i forändring. Om utvecklingen fram till EU-medlemskapet. Kristianstad: Sober förlag

Törnros, J. (1995): Effects oF a Random Breath Testing Campaign in Southern Sweden. In: Proceedings of the 13th International Conference on Alcohol, Drugs and Traffic Safety. Adelaide, August 13-18

Zador, P.L. (1991): Alcohol-Related Relative Risk of Fatal Driver Injuries in Relation to Driver Age and Sex. Journal of Studies on Alcohol 52 (4) : 302-310.

Åberg, L. (1986) : Effekter av utandningsprov på objektiv och subjektiv upptäcktsrisk. Transportforskningsberedningens rapport 1986:12, Stockholm

Öström, M. \& Eriksson, A. (1993): Single Vehicle Crashes and Alcohol: A Retrospective Study of Fatalities in Northern Sweden. Accident analysis and prevention 25 (2): 171-176

Öström, M. \& Eriksson, A. \& Björnstig, U.(1993): Reliability of Swedish alcohol statistics in traffic fatalities. A comparison between official statistics and blood alcohol analysis. Journal of traffic medicine 21 (4): 171-176. 